\title{
Human action recognition using time delay input radial basis function networks
}

\begin{abstract}
This paper presents a fast, vision-based method for the problem of human action representation and recognition. The first problem is addressed by constructing an action descriptor from spatiotemporal data of action silhouettes based on appearance and motion features. For action classification, a new Radial Basis Function Network (RBF), called Time Delay Input Radial Basis Function Network (TDIRBF) is proposed by introducing time delay units to the RBF in a novel approach. A TDIRBF offers a few desirable features such as an easier learning process and more flexibility. The representational power and speed of the proposed method were explored using a publicly available dataset. Based on experimental results, implemented in MATLAB and on standard PCs, the average time for constructing a feature vector for a high-resolution video was just about $20 \mathrm{~ms} /$ frame (or $50 \mathrm{fps}$ ) and the classifier speed was above 15 fps. Furthermore, the proposed approach demonstrated good performance in terms of both execution time and overall performance (a new performance measure that combines accuracy and speed into one metric).
\end{abstract}

Keyword: Action recognition; Action representation; Motion descriptor; Neural network; Radial basis function network 